\title{
Adjusting An Existing Forecasting Model When Some Future Demands Are Known In Advance: A Bayesian Technique
}

Raida Abuizam, (Email: abuizam@calumet.purdue.edu), Purdue University Calumet Nick T. Thomopoulos, (Email: thomop@stuart.iit.edu), Illinois Institute of Technology

\begin{abstract}
The purpose of this research is to provide a model which can be used to adjust forecasts that are already available. It analyzes the components of the advanced demand, namely, the number of orders and their corresponding order size. It explores and analyzes the possibility of using the expected number of orders for a future period as the variable to be estimated. The Bayesian estimate of the expected number of orders is used in determining the adjusted forecast. A simulation is applied to calculate a ratio between the adjusting forecasting error and the original forecasting error. Results prove that the adjusted forecast provides greater accuracy for different probable values of getting an order in advance.
\end{abstract}

\section{INTRODUCTION}

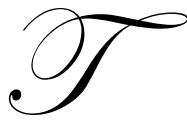

here are many sources of information on forecasting methods from simple and naïve to more sophisticated and complex. In recent years, there have been effective developments and progress in the forecasting field. Chambers, Mullick and Smith [1971] presented a study to determine how to select the most appropriate forecasting technique. Later Makridakis, Wheelwright and McGee [1984] summarized and presented a categorical breakdown of the forecasting methods and combined them with real world applications. Also, Georgoff and Murdick [1986] developed tables relating forecasting techniques to different variables including forecast horizon, time span, cost, data available, nature of product and services, accuracy, and impulse response. The detailed explanation of how to employ time-series methods is the topic of several texts. With the exception of one section in Thomopoulos' text [1980] and Warren [1981], none of the sources make any attempt to adjust the forecast when some future demands are known in advance.

All time-series forecasting methods depend particularly on past sales data. The opinion or information about what a customer is going to do is not generally part of the forecasting algorithm. Once a time-series forecast is identified, little is done to change it until more sales data are known. Sometimes, more information about sales for a period becomes available after the forecast is determined. This "advance" information does not find its way into the forecast, because these are not yet sales. The following are some of these advance demand information:

- $\quad$ An order of 500 units is received, with 100 to be shipped each month for the next five months.

- A sales representative gets an order of 1000 parts, but, due to logistic problems, has requested a delay of two to four months in shipment.

- $\quad$ The production department receives a long awaited component needed to complete a back order. They have sent word to all their intent to fill a back order.

- $\quad$ The sales representative for a region receives advance notice that a customer planning to go to a second shift any time within three months. He/She has been approached to increase the shipment of an essential part in the operation. 
- $\quad$ The salesperson may notify the forecaster in March that he/she has a pending order for thirty units from a particular customer, but the customer cannot activate the order until the coming June for some reasons.

In this fashion the forecaster has more available information about the demands for a given period. The question is how to incorporate this information into the forecast. Usually the forecaster has produced a forecast for that time period without the benefit of the future demand information. The question then becomes how the original forecast is being altered to reflect the new information.

The model presented in this paper does not update the original forecast, but adjusts the forecasted value so as to reflect the new information. Any adjustment of the original model should be done according to the procedure described in that model. The objective of the adjusted forecast is to lower the forecasting error for the period on the question. More specifically, the intent is to lower the standard deviation of the forecasting error.

Improving the forecast is an important aspect to planning and control. It can be shown that given a desired service level, lead time, and behavior of sales, that a $10 \%$ reduction in the standard forecasting errors can reduce the safety stock by as much as $17 \%$. For these items that do not require a safety stock, decreasing the forecasting error aids in budget control, cash flow, and short range planning. An improved forecast is one that produces values that show less deviation from the true value than the previous model.

\section{METHODOLOGY}

In this section, the characteristics of the binomial probability distribution, the Poisson probability distribution, the normal probability distribution and the properties of Bayes' theorem will be used to develop a model that can be used to adjust forecasts that are already available. It is assumed that the already available forecasts have been generated without any use of the information known on advanced demand.

\section{MATHEMATICAL MODEL DEVELOPMENT}

Given:

$X_{t} \quad$ The demand based on historical data, where $\mathrm{t}=1, \ldots ., \mathrm{T}$

$\mathrm{T}$ The most current time period

$n_{t} \quad$ The history of the number of orders for each time period $t$

$\bar{d} \quad$ The average order size, where $\bar{d}=\frac{\sum_{t=1}^{T} X_{t}}{\sum_{t=1}^{T} n_{t}}$

The forecaster can generate $\hat{X}_{T}(\tau)$ which is a forecasting technique that best fit the data and $\sigma_{x}$ which is the standard deviation of demand. $\hat{X}_{T}(\tau)$ can be generated using horizontal model, trend model, seasonal model, or any appropriate forecasting model that best fit the historical data.

Suppose that at a current time period $\mathrm{T}$, information becomes known about some advanced number of orders scheduled for $\mathrm{t}=\mathrm{T}+\tau$ and their corresponding order size as follows:

$n_{a \tau} \quad$ The number of advanced orders in future period $\tau$ as of time $\square \mathrm{T}$

$d_{i a \tau}$ The advanced number of units in the $\mathrm{i}^{\text {th }}$ order for period $\mathrm{T}+\tau$ 
$D_{a \tau} \quad$ The total units of advanced demand for future period $\tau \square \square$ where

$D_{a \tau}=d_{1 a \tau}+\ldots+d_{n a \tau}$

The forecast for period $\tau$ is adjusted as follows:

$\tilde{X}_{T}(\tau)=D_{a \tau}+F_{R \tau}$

where:

$\tilde{X}_{T}(\tau)$ The adjusted forecast for future period $\tau$ as of time $\mathrm{T}$

$F_{R \tau}$ The forecast for the remainder portion of period $\tau$

Defining $F_{R \tau}=n_{R \tau} \bar{d}$

where:

$n_{R \tau} \quad$ The estimate of the number of remaining orders at time $\tau$

$\bar{d} \quad$ The average order size

Substituting $F_{R \tau}$ with its value in Equation (1) will structures the following formula:

$\tilde{X}_{T}(\tau)=D_{a \tau}+n_{R \tau} \bar{d}$

If $n_{\tau}$ is defined to be the expected number of orders for future period $\square \square \square$ then:

$n_{\tau}=n_{a \tau}+n_{R \tau}$

Therefore, the value of $n_{R \tau}$ is calculated as follows:

$n_{R \tau}=n_{\tau}-n_{a \tau}$

where: $n_{R \tau} \geq 0$

The value of $n_{\tau}$ should be estimated. Substituting the value of $n_{R \tau}$ in Equation (2) structures the following equations:

$\tilde{X}_{T}(\tau)=D_{a \tau}+\left(n_{\tau}-n_{a \tau}\right) \bar{d}$

$\tilde{X}_{T}(\tau)=D_{a \tau}+n_{\tau} \bar{d}-n_{a \tau} \bar{d}$

To estimate the expected number of orders for future period $\tau$, denoted by $n_{\tau}$, the appropriate estimate of the probability distribution of $n_{\tau}$ must be found. The Poisson probability distribution is used if the estimate of the mean 
number of orders is less than ten. On the other hand, the normal probability distribution is used when the mean number of orders is greater than or equal to ten.

The mean number of orders is based on the forecast for that period without any knowledge of the advanced demand. Therefore, the estimate of the mean number of orders is equal to the forecast divided by the average order size.

$\hat{n}_{\tau}=\frac{\hat{X}_{T}(\tau)}{\bar{d}}$

where:

$\hat{n}_{\tau} \quad$ The estimate of the mean number of orders for future period $\tau$

$\hat{X}_{T}(\tau) \quad$ The forecast for future period $\tau$ as of time $\mathrm{T}$

$\bar{d} \quad$ The average order size

The standard deviation of the number of orders in future period $\tau$ is equal the standard deviation of demand divided by the average order size.

$$
\hat{\sigma}_{n}=\frac{\sigma_{x}}{\bar{d}}
$$

where:

$\hat{\sigma}_{n} \quad$ The estimate of the standard deviations of the number of orders

$\sigma_{x} \quad$ The standard deviation of demand

$\bar{d} \quad$ The average order size

To estimate the expected number of orders in future period $\tau \square$ denoted by $n_{\tau}$, the following procedure will be used:

- $\quad$ Estimate of the distribution of the number of orders $n$ and their corresponding probabilities $\mathrm{P}(\mathrm{n})$.

The Poisson probability distribution will be a good approximate to the number of orders $\mathrm{n}$ when the mean number of orders is less than ten. The mathematical formula for the Poisson probability distribution is as follows:

$$
P(n)=\frac{\left(\hat{n}_{\tau}\right)^{n} e^{-\hat{n}_{\tau}}}{n !}
$$

The normal probability distribution is used to estimate the number of orders when the mean number of orders is greater than or equal to ten.

- $\quad$ Estimate of the probability of an order in future period $\tau$ is called in advance.

The symbol $\theta_{\tau}$ is used to represent the probability an order in future period $\tau$ is called in advance $\square \square \square$ For simplicity, three estimate values of $\square \theta_{\tau}$ are used in this study, $\square \square$ namely, $\theta_{1}, \square \theta_{2}$, and $\theta_{3}$. In the mean time, the probability of occurrences for $\theta_{i}$ should be estimated. The sum of $\mathrm{P}\left(\theta_{i}\right)$ has to equal one. 
- $\quad$ Estimate of the conditional probability of advanced number of orders for each combination of $n_{j}$ and $\theta_{i}$.

$$
\begin{aligned}
& P\left\langle n_{a \tau} \mid n_{j}, \theta_{i}\right\rangle \text { is calculated using the binomial probability distribution as follows: } \\
& P\left\langle n_{a \tau} \mid n_{j}, \theta_{i}\right\rangle={ }_{n_{j}} C_{n_{a \tau}} \theta_{i}^{n_{a \tau}}\left(1-\theta_{i}\right)^{n_{j}-n_{a \tau}}
\end{aligned}
$$

- $\quad$ Estimate of the marginal probability.

The marginal probability $P\left\langle n_{a \tau} \mid n_{j}\right\rangle$ is calculated as follows:

$$
P\left\langle n_{a \tau} \mid n_{j}\right\rangle=\sum_{i=1}^{3} P\left\langle n_{a \tau} \mid n_{j}, \theta_{i}\right\rangle P\left\langle\theta_{i}\right\rangle
$$

- $\quad$ Estimate of the probability of the number of orders given the advanced number of orders.

Bayes' Theorem is used to calculate $P\left\langle n_{j} \mid n_{a \tau}\right\rangle$ as follows:

$$
P\left\langle n_{j} \mid n_{a \tau}\right\rangle=\frac{P\left\langle n_{a \tau} \mid n_{j}\right\rangle p\left\langle n_{j}\right\rangle}{p\left\langle n_{a \tau}\right\rangle}
$$

where:

$$
P\left\langle n_{a \tau}\right\rangle=\sum_{j} P\left\langle n_{a \tau} \mid n_{j}\right\rangle p\left\langle n_{j}\right\rangle
$$

- $\quad$ Estimate of the expected number of orders in future period $\tau\left(n_{\tau}\right)$.

The expected value of $n_{j}$ given $n_{a \tau}$ is now calculated as follows, knowing that this is a Bayesian estimate of n:

$$
\begin{aligned}
& E\left\langle n \mid n_{a \tau}\right\rangle=\sum_{j} n_{j} P\left\langle n_{j} \mid n_{a \tau}\right\rangle \\
& E\left\langle n \mid n_{a \tau}\right\rangle=n_{\tau}
\end{aligned}
$$

\section{AN EMPIRICAL EXAMPLE}

Consider a situation where at the current time period $\mathrm{T}$, the forecaster learns that there are two advance orders scheduled for $\mathrm{t}=\mathrm{T}+\tau$, where $\tau=3$, with corresponding order sizes of 91 and 100, respectively. Given the current forecast for time $\mathrm{T}+\tau$ is equal to $\hat{X}_{T}(\tau)=467.33$ units. Adjustment in the forecast as of time $\mathrm{T}$ is needed in lieu of the advanced knowledge of demand. The forecast adjustment procedure will be as follows:

Let $\hat{n}_{\tau}=\frac{\hat{X}_{T}(\tau)}{\bar{d}}$

where: 
$\hat{n}_{\tau} \quad$ represents the estimate of the mean number of orders for future period $\tau$

$\hat{X}_{T}(\tau)$ represents the forecast for future period $\tau \square$ as of time $\mathrm{T}$

$\bar{d} \quad$ represents the average order size

In this example, $\bar{d}=\frac{\sum_{t=1}^{T} X_{t}}{\sum_{t=1}^{T} n_{t}}=\frac{5608}{63}=89.01$ units.

Therefore, $\hat{n}_{3}=\frac{\hat{X}_{12}(3)}{\bar{d}}=\frac{467.33}{89.01}=5.25$ orders

Since the estimate of the mean number of orders $\hat{n}_{3}$ is less than ten, the Poisson probability distribution is used to estimate the number of orders $\mathrm{n}$. The values of $\mathrm{n}$ should be greater than or equal $n_{a \tau}$, therefore $\mathrm{n} \geq 2$. In this case, the probability of $\mathrm{n}$ will be truncated for $n \geq 2$. The sum of $\mathrm{P}(\mathrm{n})$ must always equal one. Tables 1 and 2 provide the steps to estimate $n_{\tau}$.

The final step presents the calculations of the adjusted forecast. After estimating $n_{\tau}$ and substituting its value in Equation (6), the estimate of the adjusted forecast is calculated as follows:

Recall that $D_{a 3}=191$ units, $\bar{d}=89.01, n_{a 3}=2$ orders, and $n_{\tau}=6.175$. Therefore,

$\tilde{X}_{T}(\tau)=D_{a \tau}+n_{\tau} \bar{d}-n_{a \tau} \bar{d}$

$\tilde{X}_{12}(3)=D_{a 3}+n_{3} \bar{d}-n_{a 3} \bar{d}$

$\tilde{X}_{12}(3)=191+6.175(89.01)-2(89.01)=592.69$ units

The adjusted forecast for the third future period is $\tilde{X}_{12}(3)=562.69$ units. This compares to the original forecast of 467.33 units 
Table 1: Steps Used To Estimate $N_{\square}$

\begin{tabular}{|c|c|c|c|c|c|}
\hline $\mathrm{n}_{\mathrm{j}}$ & $P\left(n_{j}\right)$ & $\begin{array}{c}\theta_{1}=0.1 \\
P\left(\theta_{1}\right)=0.2 \\
P\left\langle n_{a \tau} \mid n_{j}, \theta_{1}\right\rangle\end{array}$ & $\begin{array}{c}\theta_{2}=0.15 \\
P\left(\theta_{2}\right)=0.5 \\
P\left\langle n_{a \tau} \mid n_{j}, \theta_{2}\right\rangle\end{array}$ & $\begin{array}{c}\theta_{3}=0.2 \\
P\left(\theta_{3}\right)=0.3 \\
P\left\langle n_{a \tau} \mid n_{j}, \theta_{3}\right\rangle\end{array}$ & $P\left\langle n_{a \tau} \mid n_{j}\right\rangle$ \\
\hline 0 & - & - & - & - & - \\
\hline 1 & - & - & - & - & - \\
\hline 2 & 0.0878 & 0.010 & 0.023 & 0.040 & 0.002 \\
\hline 3 & 0.1463 & 0.027 & 0.057 & 0.096 & 0.009 \\
\hline 4 & 0.1829 & 0.049 & 0.098 & 0.154 & 0.019 \\
\hline 5 & 0.1829 & 0.073 & 0.138 & 0.205 & 0.026 \\
\hline 6 & 0.1524 & 0.098 & 0.176 & 0.246 & 0.027 \\
\hline 7 & 0.1089 & 0.124 & 0.210 & 0.275 & 0.023 \\
\hline 8 & 0.0680 & 0.149 & 0.238 & 0.294 & 0.016 \\
\hline 9 & 0.0378 & 0.172 & 0.260 & 0.302 & 0.009 \\
\hline 10 & 0.0189 & 0.194 & 0.276 & 0.302 & 0.005 \\
\hline 11 & 0.0086 & 0.213 & 0.287 & 0.295 & 0.002 \\
\hline 12 & 0.0036 & 0.230 & 0.292 & 0.283 & 0.000 \\
\hline 13 & 0.0014 & 0.245 & 0.294 & 0.268 & 0.000 \\
\hline 14 & 0.0005 & 0.257 & 0.291 & 0.250 & 0.000 \\
\hline 15 & 0.0002 & 0.267 & 0.286 & 0.231 & 0.000 \\
\hline 16 & 0.0000 & 0.275 & 0.277 & 0.211 & 0.000 \\
\hline
\end{tabular}

Table 2: Steps Used To Estimate $N_{\square \square}$ (Continued)

\begin{tabular}{|c|c|c|c|}
\hline $\mathrm{n}_{\mathrm{j}}$ & $P\left\langle n_{a \tau} \mid n_{j}\right\rangle$ & $P\left\langle n_{j} \mid n_{a \tau}\right\rangle$ & $n_{j} P\left\langle n_{j} \mid n_{a \tau}\right\rangle$ \\
\hline 0 & - & - & - \\
\hline 1 & - & - & 0.0311 \\
\hline 2 & 0.002 & 0.0646 & 0.1937 \\
\hline 3 & 0.009 & 0.1342 & 0.5367 \\
\hline 4 & 0.019 & 0.1862 & 1.1644 \\
\hline 5 & 0.026 & 0.1941 & 1.1345 \\
\hline 6 & 0.027 & 0.1621 & 0.9037 \\
\hline 7 & 0.023 & 0.1130 & 0.6083 \\
\hline 8 & 0.016 & 0.0676 & 0.3544 \\
\hline 10 & 0.009 & 0.0354 & 0.1820 \\
\hline 11 & 0.005 & 0.0165 & 0.0835 \\
\hline 12 & 0.002 & 0.0070 & 0.0347 \\
\hline 13 & 0.000 & 0.0027 & 0.0131 \\
\hline 15 & 0.000 & 0.0009 & 0.0046 \\
\hline 16 & 0.000 & 0.0003 & 0.0000 \\
\hline
\end{tabular}




\section{FORECASTING ERROR ANALYSIS}

This section illustrates the application of the Bayesian model presented in this paper. Since the model is not explored, the distributions of the future demand data is also unexplored. No source is available to suggest the prevailing status of this data. It is the lack of exploration that prompted the use of simulation to test the accuracy and the range of applicability for the model. By following the simulation process numerically, the forecaster will be able to compare the ratio of the adjusted forecasting error to the original forecasting error denoted by $R a$ using different parameters with different values of $\theta$.

If $R a<1 \Rightarrow$ the adjusted forecast provides greater accuracy.

If $R a>1 \Rightarrow$ the original forecast provides greater accuracy.

If $R a=1 \quad \Rightarrow$ no improvements occurs.

Table 3 provides values of $R a$ based on different parameters of $\mu_{n}, \sigma_{n}$, and $\theta$. Where: $R a=\frac{\sum_{i=1}^{N}\left|e_{a i}\right|}{\sum_{i=1}^{N}\left|e_{i}\right|}$

$R a$ represents a ratio measure

$e_{a i}$ represents the adjusted forecasting error

$e_{i}$ represents the original forecasting error

| | represents finding the absolute value

$N$ represents the number of trials

$\mu_{n}$ represents the mean number of orders

$\sigma_{n}$ represents the standard deviation of the number of orders

$\theta$ represents the probability of getting an order in advance

The calculations in Table 3 are based on thirty trials.

For further analysis, figures are drawn to show the behavior of $R a$ versus the mean number of orders $\mu_{n}$ for different values of $\theta \square \square \square$ Figure 1 provides the values of $R a$ versus $\mu_{n}$ for all values of $\theta$. Figure 2 presents the behavior of $R a$ versus $\theta$ for different values of $\mu_{n}$. 
Figure 1: Values of $R a$ Versus $\mu_{n}$ For All Values Of $\theta$

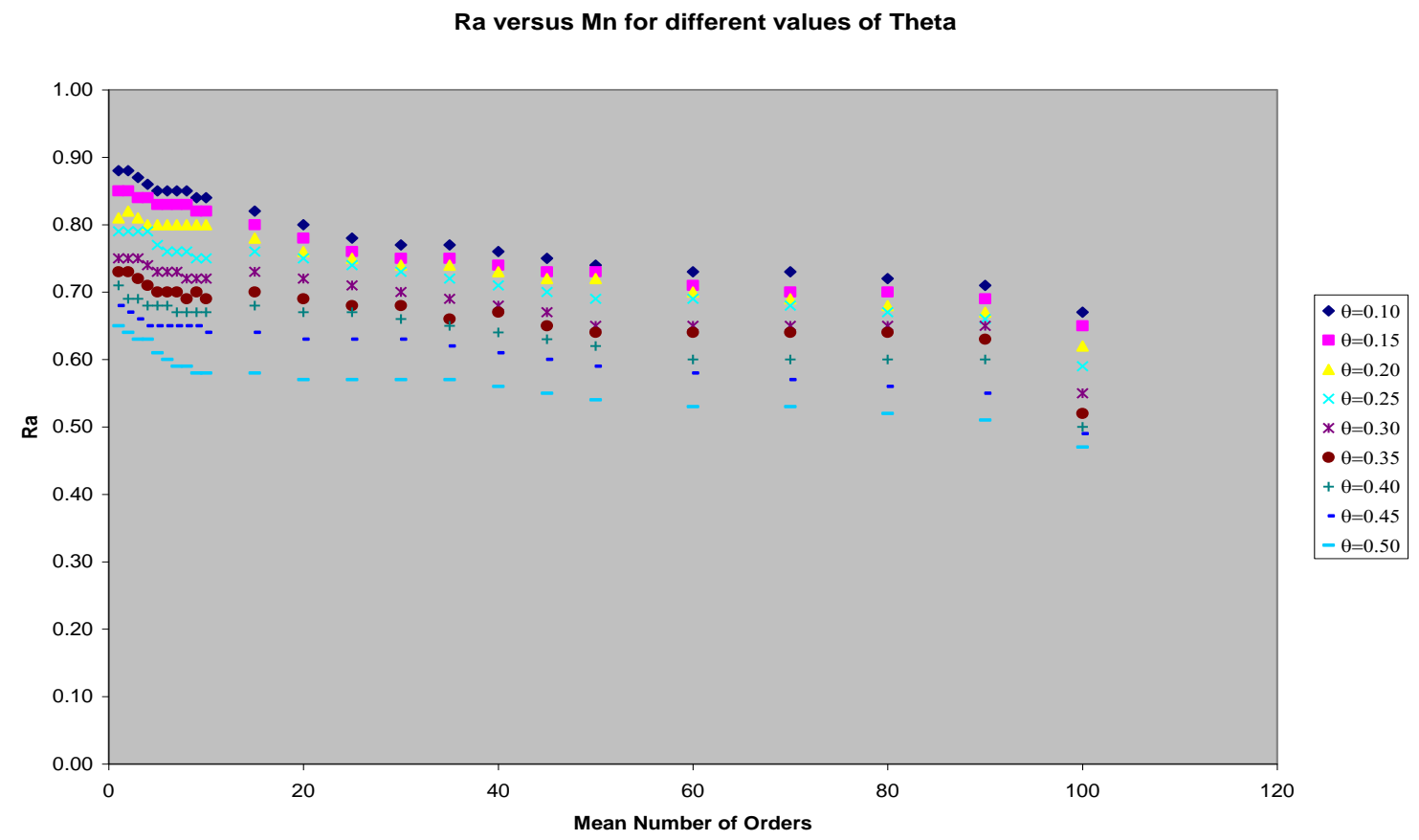

Table 3: Values of $R a$

\begin{tabular}{|c|c|c|c|c|c|c|c|c|c|c|}
\hline$\square_{n}$ & $\square_{n}$ & & & & & $\square$ & & & & \\
\hline & & 0.10 & 0.15 & 0.20 & 0.25 & 0.30 & 0.35 & 0.40 & 0.45 & 0.50 \\
\hline & & & & & & & & & & \\
\hline 1 & 0.30 & 0.88 & 0.85 & 0.81 & 0.79 & 0.75 & 0.73 & 0.71 & 0.68 & 0.65 \\
\hline 2 & 0.50 & 0.88 & 0.85 & 0.82 & 0.79 & 0.75 & 0.73 & 0.69 & 0.67 & 0.64 \\
\hline 3 & 0.90 & 0.87 & 0.84 & 0.81 & 0.79 & 0.75 & 0.72 & 0.69 & 0.66 & 0.63 \\
\hline 4 & 1.00 & 0.86 & 0.84 & 0.80 & 0.79 & 0.74 & 0.71 & 0.68 & 0.65 & 0.63 \\
\hline 5 & 1.00 & 0.85 & 0.83 & 0.80 & 0.77 & 0.73 & 0.70 & 0.68 & 0.65 & 0.61 \\
\hline 6 & 1.50 & 0.85 & 0.83 & 0.80 & 0.76 & 0.73 & 0.70 & 0.68 & 0.65 & 0.60 \\
\hline 7 & 2.00 & 0.85 & 0.83 & 0.80 & 0.76 & 0.73 & 0.70 & 0.67 & 0.65 & 0.59 \\
\hline 8 & 2.50 & 0.85 & 0.83 & 0.80 & 0.76 & 0.72 & 0.69 & 0.67 & 0.65 & 0.59 \\
\hline 9 & 3.00 & 0.84 & 0.82 & 0.80 & 0.75 & 0.72 & 0.70 & 0.67 & 0.65 & 0.58 \\
\hline 10 & 3.00 & 0.84 & 0.82 & 0.80 & 0.75 & 0.72 & 0.69 & 0.67 & 0.64 & 0.58 \\
\hline 15 & 4.00 & 0.82 & 0.80 & 0.78 & 0.76 & 0.73 & 0.70 & 0.68 & 0.64 & 0.58 \\
\hline 20 & 5.00 & 0.80 & 0.78 & 0.76 & 0.75 & 0.72 & 0.69 & 0.67 & 0.63 & 0.57 \\
\hline 25 & 5.00 & 0.78 & 0.76 & 0.75 & 0.74 & 0.71 & 0.68 & 0.67 & 0.63 & 0.57 \\
\hline 30 & 10.00 & 0.77 & 0.75 & 0.74 & 0.73 & 0.70 & 0.68 & 0.66 & 0.63 & 0.57 \\
\hline 35 & 10.00 & 0.77 & 0.75 & 0.74 & 0.72 & 0.69 & 0.66 & 0.65 & 0.62 & 0.57 \\
\hline 40 & 12.00 & 0.76 & 0.74 & 0.73 & 0.71 & 0.68 & 0.67 & 0.64 & 0.61 & 0.56 \\
\hline 45 & 15.00 & 0.75 & 0.73 & 0.72 & 0.70 & 0.67 & 0.65 & 0.63 & 0.60 & 0.55 \\
\hline 50 & 15.00 & 0.74 & 0.73 & 0.72 & 0.69 & 0.65 & 0.64 & 0.62 & 0.59 & 0.54 \\
\hline 60 & 20.00 & 0.73 & 0.71 & 0.70 & 0.69 & 0.65 & 0.64 & 0.60 & 0.58 & 0.53 \\
\hline 70 & 20.00 & 0.73 & 0.70 & 0.69 & 0.68 & 0.65 & 0.64 & 0.60 & 0.57 & 0.53 \\
\hline 80 & 25.00 & 0.72 & 0.70 & 0.68 & 0.67 & 0.65 & 0.64 & 0.60 & 0.56 & 0.52 \\
\hline 90 & 30.00 & 0.71 & 0.69 & 0.67 & 0.66 & 0.65 & 0.63 & 0.60 & 0.55 & 0.51 \\
\hline 100 & 30.00 & 0.67 & 0.65 & 0.62 & 0.59 & 0.55 & 0.52 & 0.50 & 0.49 & 0.47 \\
\hline
\end{tabular}


Figure 2: Values Of $R a$ Versus $\theta \square$ For Different Values Of $\mu_{n}$

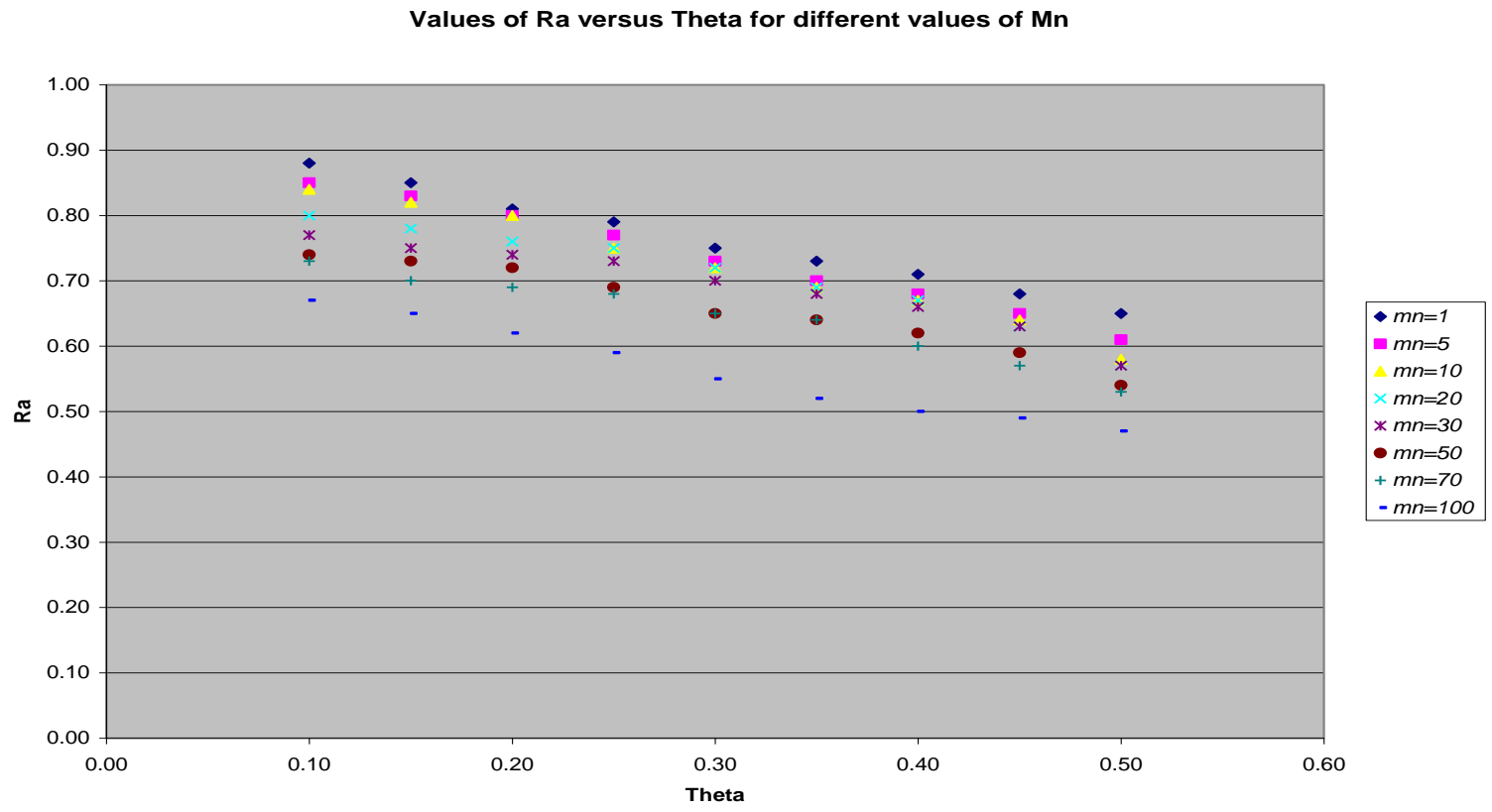

Figure 1 shows that the values of $R a$ decrease as the mean number of orders increase. The Bayesian model uses the normal distribution as an approximate to the number of orders $n$ when the mean number of orders is greater than or equal to ten. The simulation results along with figure 1 prove that the use of the normal distribution as an estimate to the number of orders leads to a more accurate adjusted forecast. Figure 2 proves that the values of $R a$ decrease as the values of $\theta \square$ increase. These figures along with the simulation results prove that knowing more information in advance leads to achieving a more accurate forecast. In the mean time, the value of the adjusted forecasting error will decrease which in turn reduces the values of $R a$.

\section{SUMMARY AND CONCLUSION}

The main objective of this paper is to present a forecasting model that adjusts an existing forecast to reflect some future demand information. Any demand consists of its two components: the number of orders and a corresponding order size for each order. The Bayesian model is developed to reflect information given in advance on part or portion of the total number of orders. Bayesian theorem is used to estimate the expected number of orders for future period $\tau$. Then the estimate of the number of remaining orders can be calculated.

To estimate the expected number of orders for future period $\tau$, it is important to find the appropriate estimate of its probability distribution. The Poisson probability distribution is used if the estimate of the mean number of orders is less than ten, while the normal probability distribution is used when the mean number of orders is greater than or equal to ten. It must be noted that the mean number of orders is based on the forecast for that period without any knowledge of the advanced number of orders.

The objective of the adjusted forecast is to lower the forecasting error for future period $\tau$. To be able to compare the adjusted forecast to the original one, the forecaster can obtain a ratio of the adjusted forecasting error to the original forecasting error. If that ratio is less than one, then the adjusted forecast provided greater accuracy. If that ratio is equal to one, then no improvement occurs. If that ratio is greater than one, then the original forecast is more accurate. 
A simulation process is used to conduct an error analysis. The purpose of this simulation is to provide in depth analysis to test the accuracy and the range of applicability of the Bayesian forecasting model presented in this paper. By following the simulation procedure numerically, the forecaster will be able to compare the ratio of the adjusted forecasting error to the original forecasting error denoted by $R a$ using different parameters with different values of $\theta$. The simulation results prove that knowing more information in advance leads to achieving more accurate forecasts. In the meantime, the value of the adjusted forecasting error will decrease which in turn reduces the values of $R a$.

\section{REFERENCES}

1. Abuizam, R. Adjusting A Forecasting Model When Some Future Demands Are Known In Advance, Unpublished ph. D. Thesis, Illinois Institute of Technology, Chicago, Illinois. 2005.

2. Brown, R. G. Smoothing, Forecasting and Prediction of Discrete Time Series. New Jersey, Prentice-Hall, Inc. 1963.

3. Chambers, J. C., Mullick, S. K., and Smith, D. D. How to Choose the Right Forecasting Technique. Harvard Business Review, Vol. 49, No. 4, pp. 45-73, 1971.

4. Georgoff, D. M. and Murdick, R. G. Manager's Guide to Forecasting. Harvard Business Review, Vol. 64, pp. 110-123, 1986.

5. Lind, D. A, Marchal W. G., and Wathen, S. A. Statistical Techniques in Business and Economics. McGraw-Hill Irwin, Burr Ridge Illinois. 2005.

6. Makridakis, S. M. and Wheelwright, S. C. Forecasting: Issues and Challenges for Marketing Accounting. Journal of Marketing, Vol. 41, No. 4, pp.24-37, 1977.

7. Thomopoulos, N. T. Applied Forecasting Methods. New Jersey, Prentice-Hall, Inc. 1980.

8. Thomopoulos, N. T. Strategic Inventory Management And Planning With Tables. Hitchcock Publishing Co. Carol Stream Illinois. 1990.

9. Warren, R. V. Forecast Modification When Some Future Demands Are Known In Advance, Unpublished ph. D. Thesis, Illinois Institute of Technology, Chicago, Illinois. 1981. 
NOTES 